\title{
On solving isomorphism problems about 2-designs using block intersection numbers
}

\author{
Christian Kaspers* and Alexander Pott*
}

November 21, 2019

\begin{abstract}
In this paper, we give a partial solution to a new isomorphism problem about 2- $(v, k, k-1)$ designs from disjoint difference families in finite fields and Galois rings. Our results are obtained by carefully calculating and bounding some block intersection numbers, and we give insight on the limitations of this technique. Moreover, we present results on cyclotomic numbers, the multiplicities of block intersection numbers of certain designs and on the structure of Galois rings of characteristic $p^{2}$.
\end{abstract}

Keywords disjoint difference family, Galois ring, combinatorial design, isomorphism problem, intersection number, cyclotomic number

\section{Introduction}

In their previous work [15], the present authors studied two constructions of difference families in Galois rings by Davis, Huczynska, and Mullen [9] and by Momihara [17]. Both constructions were inspired by a classical construction of difference families in finite fields which was introduced by Wilson [21] in 1972. Various types of difference families have long been studied in combinatorial literature $[1,3,5,6,7,12,14,21]$. They have applications in coding theory and communications and information security [18], and they are related to many other combinatorial objects. In particular, every difference family gives rise to a combinatorial design. Combinatorial designs themselves have been extensively studied since the first half of the 19th century, they have many applications in group theory, finite geometry and cryptography $[3,8]$.

Whenever a new construction of difference families is given, the natural question arises whether the associated designs are also new or whether they are isomorphic to known designs. By calculating and bounding some block intersection numbers, the present authors [15] solved this isomorphism problem for the difference families from

\footnotetext{
*Institute for Algebra and Geometry, Otto von Guericke University Magdeburg, 39106 Magdeburg, Germany (email: christian.kaspers@ovgu.de, alexander.pott@ovgu.de)
} 
Momihara [17] and Wilson [21] and for those from Davis, Huczynska, and Mullen [9] and Wilson [21]. In this paper, we obtain new difference families from the ones constructed by Davis, Huczynska, and Mullen [9]. These new difference families also have an analogue in finite fields from Wilson's [21] construction. Motivated by the present authors' previous results, we will use the same technique as in their paper [15] to study whether the associated designs are isomorphic or not. It will become clear that the approach to use block intersection numbers as a tool to solve isomorphism problems is promising for certain types of designs but has its limitations in general.

We start by defining the objects we study in this paper. First, we need the following notations: Let $G$ be an additively written abelian group, $A, B \subseteq G$ and $g \in G$. We define multisets

$$
\begin{aligned}
\Delta A & =\left\{a-a^{\prime}: a, a^{\prime} \in A, a \neq a^{\prime}\right\}, \\
A-B & =\{a-b: a \in A, b \in B, a \neq b\}, \\
A+g & =\{a+g: a \in A\} .
\end{aligned}
$$

We will sometimes use these notations to denote sets, not multisets. It will be clear from the context whether we mean the multiset or the respective set.

Definition 1. Let $G$ be an abelian group of order $v$, and let $D_{1}, D_{2}, \ldots, D_{b}$ be $k$-subsets of $G$. The collection $D=\left\{D_{1}, D_{2}, \ldots, D_{b}\right\}$ is called a $(v, k, \lambda)$ difference family in $G$ if each nonzero element of $G$ occurs exactly $\lambda$ times in the multiset union

$$
\bigcup_{i=1}^{b} \Delta D_{i} \text {. }
$$

If the subsets $D_{1}, D_{2}, \ldots, D_{b}$ are mutually disjoint, they form a disjoint difference family. If $b=1$, one speaks of a $(v, k, \lambda)$ difference set. We call $D$ near-complete if the subsets $D_{1}, D_{2}, \ldots, D_{b}$ partition $G \backslash\{0\}$.

In this paper, we focus on near-complete $(v, k, k-1)$ disjoint difference families. For more background on this type of difference families, the reader is referred to the survey by Buratti [5] who summarizes many results and introduces a powerful new construction. His construction includes many known constructions, including the one by Davis, Huczynska, and Mullen [9]. However, it seems to be too general to use it for studying isomorphism problems, at least when using block intersection numbers. Eventually, we remark that every near-complete disjoint difference family is also an external difference family $[7,9,15]$.

As mentioned above, every difference family gives rise to a combinatorial design.

Definition 2. Let $P$ be a set with $v$ elements that are called points. A $t-(v, k, \lambda)$ design, or $t$-design, in brief, is a collection of $k$-subsets, called blocks, of $P$ such that every $t$-subset of $P$ is contained in exactly $\lambda$ blocks.

The associated designs of difference families are 2-designs which are often referred to as balanced incomplete block designs (BIBD). They are constructed as the development of a difference family. 
Definition 3. Let $G$ be an abelian group, and let $D=\left\{D_{1}, D_{2}, \ldots, D_{b}\right\}$ be a collection of subsets of $G$. The development of $D$ is the collection

$$
\operatorname{dev}(D)=\left\{D_{i}+g: D_{i} \in D, g \in G\right\}
$$

of all the translates of the sets $D_{1}, D_{2}, \ldots, D_{b}$. The sets $D_{1}, D_{2}, \ldots, D_{b}$ are called the base blocks of $\operatorname{dev}(D)$.

In other words, the development of $D$ is the union of the orbits of the sets contained in $D$ under the action of $G$. If all orbits have full length, $\operatorname{dev}(D)$ contains $v b$ blocks. The following well-known proposition relates difference families to 2-designs.

Proposition 1.1. Let $D$ be a $(v, k, \lambda)$ difference family in an abelian group $G$. The development $\operatorname{dev}(D)$ of $D$ forms a $2-(v, k, \lambda)$ design with point set $G$.

\section{Galois rings}

In this section, we give a short introduction to Galois rings and present some of their well-known properties needed in this paper. We refer to the work by McDonald [16] and Wan [20] for extended general background on this topic. Let $p$ be a prime, and let $f(x) \in \mathbb{Z}_{p^{m}}[x]$ be a monic basic irreducible polynomial of degree $r \geq 1$, which means that the image of $f$ modulo $p$ in $\mathbb{F}_{p}[x]$ is irreducible. The factor ring

$$
\mathbb{Z}_{p^{m}}[x] /\langle f(x)\rangle
$$

is called a Galois ring of characteristic $p^{m}$ and extension degree $r$. It is denoted by $\operatorname{GR}\left(p^{m}, r\right)$, and its order is $p^{m r}$. Since any two Galois rings of the same characteristic and order are isomorphic, we will speak of the Galois ring $\operatorname{GR}\left(p^{m}, r\right)$.

Galois rings are local commutative rings. The unique maximal ideal of the ring $R=$ $\operatorname{GR}\left(p^{m}, r\right)$ is

$$
\mathcal{I}=p R=\{p a: a \in R\} .
$$

The factor ring $R / \mathcal{I}$ is isomorphic to the finite field $\mathbb{F}_{p^{r}}$ with $p^{r}$ elements. As a system of representatives of $R / \mathcal{I}$, we take the Teichmüller set

$$
\mathcal{T}=\left\{0,1, \xi, \ldots, \xi^{p^{r}-2}\right\}
$$

where $\xi$ denotes a root of order $p^{r}-1$ of $f(x)$. It is convenient to choose the generalized Conway polynomial, that is the Hensel lift from $\mathbb{F}_{p}[x]$ to $\mathbb{Z}_{p^{m}}[x]$ of the Conway polynomial, as our polynomial $f(x)$. Then, $x+\langle f\rangle$ is a generator of the Teichmüller group, and we set $\xi=x+\langle f\rangle$. Zwanzger [22, Section 1.3] provides more information on the generalized Conway polynomial and its construction. Every $a \in R$ has a unique $p$-adic representation $a=\alpha_{0}+p \alpha_{1}+\cdots+p^{m-1} \alpha_{m-1}$, where $\alpha_{0}, \alpha_{1}, \ldots, \alpha_{m-1} \in \mathcal{T}$.

The elements of $R \backslash \mathcal{I}$ are all the units of $R$. We denote this unit group by $R^{*}$. It has order $p^{m r}-p^{(m-1) r}$ and is the direct product of the cyclic Teichmüller group

$$
\mathcal{T}^{*}=\mathcal{T} \backslash\{0\}
$$


of order $p^{r}-1$ and the group of principal units $\mathbb{P}=1+\mathcal{I}$ of order $p^{(m-1) r}$. If $p$ is odd or if $p=2$ and $m \leq 2$, then $\mathbb{P}$ is a direct product of $r$ cyclic groups of order $p^{m-1}$. If $p=2$ and $m \geq 3$, then $\mathbb{P}$ is a direct product of a cyclic group of order 2 , a cyclic group of order $2^{m-2}$, and $r-1$ cyclic groups of order $2^{m-1}$. In this paper, we will only consider Galois rings of characteristic $p^{2}$. In this case, $(1+p \alpha)(1+p \beta)=1+p(\alpha+\beta)$ for any $\alpha, \beta \in \mathcal{T}$, and every unit $u \in \operatorname{GR}\left(p^{2}, r\right)^{*}$ has a unique representation

$$
u=\alpha_{0}\left(1+p \alpha_{1}\right),
$$

where $\alpha_{0} \in \mathcal{T}^{*}$ and $\alpha_{1} \in \mathcal{T}$. Moreover, the group of principal units $\mathbb{P}$ is a direct product of $r$ cyclic groups of order $p$ and thus has the structure of an elementary abelian group of order $p^{r}$.

\section{Construction of disjoint difference families}

In this section, we describe three constructions of disjoint difference families. The constructions from Theorem 3.2 and Theorem 3.3 are well known. The third construction, in Theorem 3.4, follows from results by Furino [12]. As the first two constructions also fall into Furino's very general framework, we will present his result first. We only restate a special case of his construction.

Theorem 3.1 ([12, Theorem 3.3 and Corollary 3.5]). Let $R$ be a commutative ring with an identity. Denote the cardinality of $R$ by $v$ and the unit group of $R$ by $R^{*}$. Let $B$ be a subgroup of $R^{*}$ of order $k$ such that $\Delta B$ is a subset of $R^{*}$. Denote by $S$ a system of representatives of the cosets of $B$ in $R \backslash\{0\}$. The collection $\{s B: s \in S\}$ is a $(v, k, k-1)$ disjoint difference family in the additive group of $R$.

Note that, by abuse of denotation, we also call sets $s B$ where $s$ is not a unit a coset of $B$. Because of the condition $\Delta B \subseteq R^{*}$, these cosets also have cardinality $k$, and all the cosets partition $R \backslash\{0\}$.

Next, we present the construction of disjoint difference families in finite fields by Wilson [21]. It makes use of the cyclotomoy of the $e$-th powers in a finite field.

Theorem 3.2. Let $\mathbb{F}_{q}$ be the finite field with $q$ elements, and let $\alpha$ be a generator of the multiplicative group $\mathbb{F}_{q}^{*}$ of $\mathbb{F}_{q}$. Moreover, let $e, f$ be integers satisfying ef $=q-1$, where $e, f \geq 2$, and let

$$
C_{i}=\left\{\alpha^{t}: t \equiv i \quad(\bmod e)\right\},
$$

where $i=0,1, \ldots, e-1$, be the cosets of the unique subgroup $C_{0}$ of index $e$ and order $f$ that is formed by the e-th powers of $\alpha$ in $\mathbb{F}_{q}^{*}$. Then, the collection $C=\left\{C_{0}, C_{1}, \ldots, C_{e-1}\right\}$ is a near-complete $(q, f, f-1)$ disjoint difference family in the additive group of $\mathbb{F}_{q}$.

We now present the construction of disjoint difference families by Davis, Huczynska, and Mullen [9]. We use the same notation as in section 2, and we remark that this theorem also follows from Theorem 3.1 and from a result by Buratti [5]. 
Theorem 3.3 ([9, Theorem 4.1]). Let $p$ be a prime, and let $r$ be a positive integer such that $p^{r} \geq 3$. Denote by $\mathcal{T}$ the Teichmüller set of the Galois ring $G R\left(p^{2}, r\right)$ and by $\mathcal{T}^{*}$ the Teichmüller group $\mathcal{T}^{*}=\mathcal{T} \backslash\{0\}$. The collection

$$
E=\left\{(1+p \alpha) \mathcal{T}^{*}: \alpha \in \mathcal{T}\right\} \cup p \mathcal{T}^{*}
$$

forms a near-complete $\left(p^{2 r}, p^{r}-1, p^{r}-2\right)$ disjoint difference family in the additive group of $G R\left(p^{2}, r\right)$.

Since $p^{r}-1$ divides $p^{2 r}-1$, there exists a disjoint difference family in the additive group of $\mathbb{F}_{p^{2 r}}$ that has the exact same parameters as the difference family from Theorem 3.3. It can be constructed using Theorem 3.2 by taking the $\left(p^{r}+1\right)$-th powers in $\mathbb{F}_{p^{2 r}}$. Inspired by Theorem 3.3 and the work by Furino [12], we noticed that if $p$ is odd, we obtain a new disjoint difference family by taking the cosets of the group of Teichmüller squares.

Theorem 3.4. Let $p$ be an odd prime and let $r$ be a positive integer such that $p^{r} \geq 5$. Moreover, let

$$
\mathcal{T}^{*}=\left\{1, \xi, \xi^{2}, \ldots, \xi^{p^{r}-2}\right\}
$$

be the Teichmüller group of the Galois ring $G R\left(p^{2}, r\right)$, and let $\mathcal{T}=\mathcal{T}^{*} \cup\{0\}$. By

$$
\mathcal{T}_{S}^{*}=\left\{1, \xi^{2}, \ldots, \xi^{p^{r}-3}\right\}
$$

we denote the set of squares and by

$$
\mathcal{T}_{N}^{*}=\left\{\xi, \xi^{3}, \ldots, \xi^{p^{r}-2}\right\}
$$

we denote the set of non-squares in $\mathcal{T}^{*}$. The collection

$$
E^{H}=\left\{(1+p \alpha) \mathcal{T}_{S}^{*}: \alpha \in \mathcal{T}\right\} \cup\left\{p \mathcal{T}_{S}^{*}\right\} \cup\left\{(1+p \alpha) \mathcal{T}_{N}^{*}: \alpha \in \mathcal{T}\right\} \cup\left\{p \mathcal{T}_{N}^{*}\right\}
$$

forms a near complete $\left(p^{2 r}, \frac{p^{r}-1}{2}, \frac{p^{r}-3}{2}\right)$ disjoint difference family in the additive group of $G R\left(p^{2}, r\right)$.

Proof. Denote by $\mathcal{I}=p \operatorname{GR}\left(p^{2}, r\right)$ the maximal ideal of $\operatorname{GR}\left(p^{2}, r\right)$. The Teichmüller set $\mathcal{T}$ is a system of representatives of $\operatorname{GR}\left(p^{2}, r\right) / \mathcal{I}$. This factor ring is isomorphic to the finite field $\mathbb{F}_{p^{r}}$. Consequently, the difference of two distinct elements of the Teichmüller group $\mathcal{T}^{*}$ is a unit, hence $\Delta \mathcal{T}^{*} \subseteq \operatorname{GR}\left(p^{2}, r\right)^{*}$. As $\mathcal{T}_{S}^{*}$ is a subgroup of $\mathcal{T}^{*}$, it follows that $\Delta \mathcal{T}_{S}^{*}$ is a subset of the unit group $\operatorname{GR}\left(p^{2}, r\right)^{*}$. In this case, according to Theorem 3.1, the collection of the cosets of $\mathcal{T}_{S}^{*}$ in $\operatorname{GR}\left(p^{2}, r\right) \backslash\{0\}$ forms a disjoint difference family in the additive group of $\operatorname{GR}\left(p^{2}, r\right)$.

Note that the difference family $E^{H}$ from Theorem 3.4 can be obtained from the difference family $E$ presented in Theorem 3.3 by cutting the base blocks of $E$ into halves, hence the name $E^{H}$. Furthermore, note that there exists a difference family $C^{H}$ in the finite field $\mathbb{F}_{p^{2 r}}$ which has the same parameters as $E^{H}$. According to Theorem 3.2, the cosets of the subgroup $C_{0}^{H}$ of the $2\left(p^{r}+1\right)$-th powers in $\mathbb{F}_{p^{2 r}}^{*}$ form a $\left(p^{2 r}, \frac{p^{r}-1}{2}, \frac{p^{r}-3}{2}\right)$ disjoint difference family in the additive group of $\mathbb{F}_{p^{2 r}}$. In the following section, we will study the isomorphism problem for the difference families $C^{H}$ and $E^{H}$ from finite fields and Galois rings. Moreover, we present additional isomorphism invariants of the designs in finite fields coming from Theorem 3.2. 


\section{A partial solution to the isomorphism problem}

Denote by $C$ the $\left(p^{2 r}, p^{r}-1, p^{r}-2\right)$ difference family and by $C^{H}$ the $\left(p^{2 r}, \frac{p^{r}-1}{2}, \frac{p^{r}-3}{2}\right)$ difference family in the additive group of $\mathbb{F}_{p^{2 r}}$ which are constructed using Theorem 3.2. Denote by $E$ the $\left(p^{2 r}, p^{r}-1, p^{r}-2\right)$ difference family and by $E^{H}$ the $\left(p^{2 r}, \frac{p^{r}-1}{2}, \frac{p^{r}-3}{2}\right)$ difference family in the additive group of $\operatorname{GR}\left(p^{2}, r\right)$ which are constructed using Theorem 3.3 and Theorem 3.4, respectively.

In their previous work, the present authors [15] solved the isomorphism problem for the 2-( $\left.p^{2 r}, p^{r}-1, p^{r}-2\right)$ designs $\operatorname{dev}(C)$ and $\operatorname{dev}(E)$. They showed that the designs are nonisomorphic for all combinations of $p$ and $r$ except $p=3$ and $r=1$. In this section, we will give a partial solution to the isomorphism problem for the 2- $\left(p^{2 r}, \frac{p^{r}-1}{2}, \frac{p^{r}-3}{2}\right)$ designs $\operatorname{dev}\left(C^{H}\right)$ and $\operatorname{dev}\left(E^{H}\right)$. Note that these designs can be obtained from $\operatorname{dev}(C)$ and $\operatorname{dev}(E)$, respectively, by cutting every block into two halves.

Remark 1. The fact that two designs $\mathcal{D}_{1}, \mathcal{D}_{2}$ are nonisomorphic does not imply that two designs $\mathcal{D}_{1}^{H}, \mathcal{D}_{2}^{H}$ that are obtained by cutting the blocks of $\mathcal{D}_{1}$ and $\mathcal{D}_{2}$ into smaller blocks are nonisomorphic. This is shown in the following example which was given in the context of skew Hadamard difference sets by Feng and Xiang [11, Example 3.3]. Denote by $C_{0}$ the subgroup of the 14-th powers of the multiplicative group of the finite field $\mathbb{F}_{11^{3}}$ and by $C_{0}, C_{1}, \ldots, C_{13}$ the cosets of $C_{0}$. It follows from Theorem 3.2 that the collection $C=\left\{C_{0}, C_{1}, \ldots, C_{13}\right\}$ is a disjoint difference family in the additive group of $\mathbb{F}_{11^{3}}$. The collections

$$
\begin{aligned}
D_{1}=\{ & \left\{C_{0} \cup C_{2} \cup C_{4} \cup C_{6} \cup C_{8} \cup C_{10} \cup C_{12}\right\}, \\
& \left.\left\{C_{1} \cup C_{3} \cup C_{5} \cup C_{7} \cup C_{9} \cup C_{11} \cup C_{13}\right\}\right\}, \\
D_{2}=\{ & \left\{C_{0} \cup C_{1} \cup C_{2} \cup C_{3} \cup C_{4} \cup C_{5} \cup C_{6}\right\}, \\
& \left.\left\{C_{7} \cup C_{8} \cup C_{9} \cup C_{10} \cup C_{11} \cup C_{12} \cup C_{13}\right\}\right\}, \\
D_{3}=\{ & \left\{C_{0} \cup C_{1} \cup C_{3} \cup C_{4} \cup C_{5} \cup C_{6} \cup C_{9}\right\}, \\
& \left.\left\{C_{2} \cup C_{7} \cup C_{8} \cup C_{10} \cup C_{11} \cup C_{12} \cup C_{13}\right\}\right\} .
\end{aligned}
$$

are also disjoint difference families in the additive group of $\mathbb{F}_{11^{3}}$. Consider their associated designs $\operatorname{dev}\left(D_{1}\right), \operatorname{dev}\left(D_{2}\right), \operatorname{dev}\left(D_{3}\right)$. Their full automorphism groups $\mathcal{A}_{1}, \mathcal{A}_{2}, \mathcal{A}_{3}$ have orders $\left|\mathcal{A}_{1}\right|=5310690,\left|\mathcal{A}_{2}\right|=252890$ and $\left|\mathcal{A}_{3}\right|=758670$. Thus, the designs are pairwise nonisomorphic. However, it is clear that from all three difference families, we can obtain the difference family $C$ by cutting their base blocks into the cyclotomic cosets $C_{0}, C_{1}, \ldots, C_{13}$. Hence, from the nonisomorphic designs $\operatorname{dev}\left(D_{1}\right), \operatorname{dev}\left(D_{2}\right), \operatorname{dev}\left(D_{3}\right)$, we can obtain the exact same design $\operatorname{dev}(C)$.

The present authors [15] solved the isomorphism problem for $\operatorname{dev}(C)$ and $\operatorname{dev}(E)$ by comparing the block intersection numbers of these designs.

Definition 4. We call an integer $N$ a block intersection number of a $t$-design $\mathcal{D}$, if $\mathcal{D}$ contains two distinct blocks $B$ and $B^{\prime}$ that intersect in $N$ elements. 
Block intersection numbers are invariant under isomorphism. For a given design $\mathcal{D}$, they can be easily computed as the entries of the matrix $M^{T} M$, where $M$ is the incidence matrix of $\mathcal{D}$ with the rows corresponding to the points and the columns corresponding to the blocks of $\mathcal{D}$. Note, however, that there also exist designs that have the exact same block intersection numbers but are nonisomorphic. One example are the designs given in Remark 1. These designs are pairwise nonisomorphic, but they all share the intersection numbers $0,332,333$. For the designs $\operatorname{dev}\left(C^{H}\right)$ and $\operatorname{dev}\left(E^{H}\right)$, however, block intersection numbers seem to distinguish the designs as the following example shows.

Example 1. The constructions from Theorem 3.2 and Theorem 3.4 yield $(625,12,11)$ disjoint difference families $C^{H}$ and $E^{H}$ in the additive groups of $\mathbb{F}_{5^{4}}$ and $\operatorname{GR}(25,2)$, respectively. The associated $2-(625,12,11)$ designs have the following block intersection numbers: for $\operatorname{dev}\left(C^{H}\right)$, they are $0,1,5,6$, and for $\operatorname{dev}\left(E^{H}\right)$, they are $0,1,2,5,6$. Hence, the two designs are nonisomorphic.

Before we start with the actual calculation of our block intersection numbers we focus on their multiplicities.

Remark 2. Not only the block intersection numbers themselves but also their multiplicities are isomorphism invariants of a combinatorial design. Hence, in the following, we will not only state the intersection numbers but also their multiplicities whenever it is possible. Although we will not use the multiplicities to solve an isomorphism problem in this paper, they might be useful for further research. To determine the multiplicity of an intersection number $N$, we will first count the number of pairs $(i, j)$ such that two blocks $B_{i}$ and $B_{j}$ intersect in $N$ elements without considering that $B_{i} \cap B_{j}=B_{j} \cap B_{i}$. In the end, we divide this number by 2 .

Example 2. The multiplicities of the block intersection numbers of the designs from Example 1 are as follows: In $\operatorname{dev}\left(C^{H}\right)$, the intersection numbers $0,1,5,6$ occur with multiplicities $410328750,117000000,195000$, and 585000, respectively. In $\operatorname{dev}\left(D^{H}\right)$, the intersection numbers 0,1,2,5,6 have multiplicities 417078 750, 100687500,10312500 , 7500 and 22500 , respectively. Hence, the multiplicities distinguish the designs.

For all three designs given in Remark 1, the multiplicities of the block intersection numbers $0,332,333$ are 1331, 2655345 and 885115, respectively. Hence, in this case, neither the intersection numbers nor their multiplicities distinguish the designs.

In the remainder of this section, we will first generally describe the block intersection numbers and their multiplicities of the designs coming from the disjoint difference families in $\mathbb{F}_{q}$ that we presented in Theorem 3.2. From this result, we will derive the block intersection numbers and their multiplicities of the designs $\operatorname{dev}(C)$ and $\operatorname{dev}\left(C^{H}\right)$. Note that the present authors, in their previous paper [15], already gave the intersection numbers of $\operatorname{dev}(C)$. We will now contribute the associated multiplicities. Finally, we will establish bounds on the intersection numbers of $\operatorname{dev}\left(E^{H}\right)$ which will lead to a partial solution of the isomorphism problem of the designs $\operatorname{dev}\left(C^{H}\right)$ and $\operatorname{dev}\left(E^{H}\right)$.

The block intersection numbers of the design from Theorem 3.2 are strongly related to the so-called cyclotomic numbers: As in Theorem 3.2, let $C_{0}, C_{1}, \ldots, C_{e-1}$ be the cosets 
of the subgroup $C_{0}$ of the $e$-th powers in $\mathbb{F}_{q}^{*}$. For fixed non-negative integers $i, j \leq e-1$, the cyclotomic number $(i, j)_{e}$ of order $e$ is defined as

$$
(i, j)_{e}=\left|\left(C_{i}+1\right) \cap C_{j}\right| .
$$

Denote by $n_{e}(N)$ the number of pairs $(i, j)$, where $i, j \leq e-1$, such that the cyclotomic number $(i, j)_{e}=N$.

Proposition 4.1. Let e, $f \geq 2$ be integers such that ef $=q-1$, and let $C$ be $a(q, f, f-1)$ disjoint difference family in the additive group of $\mathbb{F}_{q}$ constructed with Theorem 3.2. The block intersection numbers of the $2-(q, f, f-1)$ design dev $(C)$ are 0 and the values of the cyclotomic numbers $(i, j)_{e}$ of order $e$ in $\mathbb{F}_{q}$. The intersection number 0 has multiplicity $\frac{1}{2} q(q-1) n_{e}(0)+\frac{1}{2} q e(e-1)$, each nonzero intersection number $N$ has multiplicity $\frac{1}{2} q(q-1) n_{e}(N)$.

Proof. Denote by $\alpha$ a primitive element of the finite field $\mathbb{F}_{q}$. Let $C=\left\{C_{0}, C_{1}, \ldots, C_{e-1}\right\}$ be a disjoint difference family from Theorem 3.2 in the additive group of $\mathbb{F}_{q}$. Take two arbitrary distinct blocks $C_{i}+a$ and $C_{j}+b$ of $\operatorname{dev}(C)$. If we want to calculate the cardinality

$$
\left|\left(C_{i}+a\right) \cap\left(C_{j}+b\right)\right|
$$

of their intersection, we need to determine the number of solutions $(s, t)$ of the equation

$$
\alpha^{s e+i}+a=\alpha^{t e+j}+b .
$$

If $a=b$, then obviously only the case $i \neq j$ is relevant. As $C_{i}$ and $C_{j}$ are disjoint, there are no solutions in this case and

$$
\left|\left(C_{i}+a\right) \cap\left(C_{j}+a\right)\right|=0 .
$$

Since there are $q$ choices for $a$ and $e(e-1)$ choices for $(i, j)$ such that $i \neq j$, the block intersection number 0 occurs $q e(e-1)$ times in this context. Removing repeated intersections, this multiplicity reduces to $\frac{q e(e-1)}{2}$.

If $a \neq b$, then $a-b=\alpha^{r}$ for some $r \in\{0, \ldots, q-1\}$. Write $r=m e+r^{\prime}$ such that $0 \leq r^{\prime} \leq e-1$. Now, we can rewrite (1) as

$$
\alpha^{(s-m) e+\left(i-r^{\prime}\right)}+1=\alpha^{(t-m) e+\left(j-r^{\prime}\right)} .
$$

Consequently,

$$
\left|\left(C_{i}+a\right) \cap\left(C_{j}+b\right)\right|=\left|\left(C_{i-r^{\prime}}+1\right) \cap C_{j-r^{\prime}}\right|,
$$

where the subscripts are calculated modulo $e$. The right-hand side of the above equation is exactly the cyclotomic number $\left(i-r^{\prime}, j-r^{\prime}\right)_{e}$. We have $q(q-1)$ choices for $(a, b)$ such that $a \neq b$, and the difference $a-b$ covers all the elements of $\mathbb{F}_{q}^{*}$ the same number of times. Consequently, each cyclotomic number $(i, j)_{e}$ that equals $N$ contributes with $q(q-1)$ to the multiplicity of the block intersection number $N$. Removing repeated intersections, this contribution reduces to $\frac{q(q-1)}{2}$. 
Using a result by Baumert, Mills, and Ward [2, Theorems 2 and 4], the present authors [15] showed that the cyclotomic numbers of order $p^{r}+1$ in $\mathbb{F}_{p^{2 r}}$ are given as

$$
\begin{aligned}
(0,0)_{p^{r}+1} & =p^{r}-2, & & \\
(0, i)_{p^{r}+1}=(i, 0)_{p^{r}+1}=(i, i)_{p^{r}+1} & =0 & & \text { for } i \neq 0, \\
(i, j)_{p^{r}+1} & =1 & & \text { for } i \neq j \text { and } i, j \neq 0 .
\end{aligned}
$$

With the help of Proposition 4.1 we can now determine the block intersection numbers of the $2-\left(p^{2 r}, p^{r}-1, p^{r}-2\right)$ design $\operatorname{dev}(C)$ and their multiplicities. While the present authors [15] presented these block intersection numbers before, the results about their multiplicities are new.

Corollary 4.2. The $2-\left(p^{2 r}, p^{r}-1, p^{r}-2\right)$ design $\operatorname{dev}(C)$ has exactly the following block intersection numbers.

\begin{tabular}{ll} 
block intersection number & multiplicity \\
\hline 0 & $\frac{1}{2}\left(3 p^{5 r}+p^{4 r}-2 p^{3 r}\right)$ \\
1 & $\frac{1}{2}\left(p^{6 r}-p^{5 r}-p^{4 r}+p^{3 r}\right)$ \\
$p^{r}-2$ & $\frac{1}{2}\left(p^{4 r}-p^{2 r}\right)$
\end{tabular}

Proof. Let $e=p^{r}+1$. Denote by $n_{e}(N)$ the number of cyclotomic numbers of order $e$ that equal $N$. In $\mathbb{F}_{p^{2 r}}$, according to (2), we have

$$
n_{e}(0)=3 p^{r}, \quad n_{e}(1)=p^{r}\left(p^{r}-1\right) \quad \text { and } \quad n_{e}\left(p^{r}-2\right)=1 .
$$

We multiply these numbers with the factor $\frac{1}{2} p^{2 r}\left(p^{2 r}-1\right)$ from Proposition 4.1. This gives us the multiplicities of the block intersection numbers 1 and $p^{r}-2$. To obtain the multiplicity for 0 , according to Proposition 4.1, we additionally need to add $\frac{1}{2} p^{2 r}\left(p^{r}+\right.$ 1) $p^{r}$.

Next, we determine the cyclotomic numbers of order $2\left(p^{r}+1\right)$ in $\mathbb{F}_{p^{2 r}}$ which are the intersection numbers of $\operatorname{dev}\left(C^{H}\right)$. Unfortunately, these parameters no longer match the conditions of the theorems by Baumert, Mills, and Ward [2] that were used to obtain the cyclotomic numbers of order $p^{r}+1$. Nevertheless, we can deduce these cyclotomic numbers from (2) with the help of the following well-known lemma.

Lemma 4.3 ([10, §67], [19, Theorem 2]). Let $p$ be an odd prime. Let $S$ be the set of nonzero squares and $N$ be the set of non-squares in the finite field $\mathbb{F}_{p^{r}}$. Denote by $Q Q$ the number of squares $s \in S$ for which $s+1$ is a nonzero square and by $Q N$ the number of $s \in S$ for which $s+1$ is not a square. Moreover, let $N N$ denote the number of nonsquares $n \in N$ for which $n+1$ is not a square and $N Q$ the number of $n \in N$ for which $n+1$ is a nonzero square.

- If $p^{r}-1 \equiv 0(\bmod 4)$, then

$$
Q Q=\frac{p^{r}-5}{4}, \quad Q N=\frac{p^{r}-1}{4}, \quad N N=\frac{p^{r}-1}{4}, \quad N Q=\frac{p^{r}-1}{4} .
$$


- If $p^{r}-1 \equiv 2(\bmod 4)$, then

$$
Q Q=\frac{p^{r}-3}{4}, \quad Q N=\frac{p^{r}+1}{4}, \quad N N=\frac{p^{r}-3}{4}, \quad N Q=\frac{p^{r}-3}{4} .
$$

Combining Lemma 4.3 with (2), we obtain the following result.

Proposition 4.4. Let $p$ be an odd prime, and let $e=p^{r}+1$ for some positive integer $r$. In $\mathbb{F}_{p^{2 r}}$, the cyclotomic numbers of order $2 e$ are as follows:

- If $p^{r}-1 \equiv 0(\bmod 4)$, then

$$
\begin{aligned}
(0,0)_{2 e} & =\frac{p^{r}-5}{4}, \\
(0, e)_{2 e}=(e, 0)_{2 e}=(e, e)_{2 e} & =\frac{p^{r}-1}{4} .
\end{aligned}
$$

- If $p^{r}-1 \equiv 2(\bmod 4)$, then

$$
\begin{aligned}
(0, e)_{2 e} & =\frac{p^{r}+1}{4}, \\
(0,0)_{2 e}=(e, 0)_{2 e}=(e, e)_{2 e} & =\frac{p^{r}-3}{4} .
\end{aligned}
$$

In both of the above cases,

$$
\begin{aligned}
(0, i)_{2 e} & =(i, 0)_{2 e}=(i, i)_{2 e}=(i, e)_{2 e} \\
& =(e, i)_{2 e}=(i, e+i)_{2 e}=(e+i, i)_{2 e}=0 \quad \text { for } i \notin\{0, e\} .
\end{aligned}
$$

Out of the remaining cyclotomic numbers,

$$
(i, j)_{2 e},(i, j+e)_{2 e},(i+e, j)_{2 e},(i+e, j+e)_{2 e}, \quad \text { where } i, j \neq 0 \text { and } i \neq j,
$$

for each choice of $i$ and $j$, exactly one cyclotomic number is 1 and the other three cyclotomic numbers are 0 , but it is not known which one is 1 .

Proof. Let $\alpha$ be a generator of $\mathbb{F}_{p^{2 r}}^{*}$, let $C_{0}$ be the unique subgroup of order $p^{r}-1$ of $\mathbb{F}_{p^{2 r}}$ formed by the $\left(p^{r}+1\right)$-th powers, and let $C_{0}, C_{1}, \ldots, C_{p^{r}}$ be the cosets of $C_{0}$. The finite field $\mathbb{F}_{p^{2 r}}$ contains a unique subfield $\mathbb{F}_{p^{r}}$ with $p^{r}$ elements. Hence, the group $C_{0}$ is the multiplicative group $\mathbb{F}_{p^{r}}^{*}$ of the subfield $\mathbb{F}_{p^{r}}$. As $p^{r}$ is odd, $C_{0}$ consists of $\frac{1}{2}\left(p^{r}-1\right)$ squares and non-squares in $\mathbb{F}_{p^{r}}$ each. Consequently,

$$
C_{0}=C_{0}^{H} \cup C_{e}^{H},
$$

where

$$
C_{0}^{H}=\left\{\alpha^{t} \mid t \equiv 0 \quad\left(\bmod 2\left(p^{r}+1\right)\right)\right\}
$$

is the set of squares and

$$
C_{e}^{H}=\left\{\alpha^{t} \mid t \equiv e \quad\left(\bmod 2\left(p^{r}+1\right)\right)\right\}
$$


is the set of non-squares in $\mathbb{F}_{p^{*}}^{*}$. The values of the cyclotomic numbers $(i, j)_{2 e}$, where $i, j \in\{0, e\}$, now follow from Lemma 4.3. In the same way as before, we can divide each of the cosets $C_{0}, C_{1}, \ldots, C_{p^{r}}$, of $C_{0}$ into two cosets $C_{i}^{H}$ and $C_{e+i}^{H}$ of $C_{0}^{H}$. Since

$$
C_{i}=C_{i}^{H} \cup C_{e+i}^{H}
$$

for all $i=0,1, \ldots, p^{r}$, we obtain

$$
\left(C_{i}+1\right) \cap C_{j}=\bigcup_{\substack{k \in\{i, e+i\} \\ \ell \in\{j, e+j\}}}\left(C_{k}^{H}+1\right) \cap C_{\ell}^{H}
$$

for $0 \leq i, j \leq p^{r}$. In terms of cyclotomic numbers, this means

$$
(i, j)_{e}=\sum_{\substack{k \in\{i, e+i\} \\ \ell \in\{j, e+j\}}}(k, \ell)_{2 e}
$$

for $0 \leq i, j \leq p^{r}$. The values of the cyclotomic numbers $(i, j)_{2 e}$, where $i, j \notin\{0, e\}$, now follow from combining (4) with (2).

Unfortunately, the exact values of the cyclotomic numbers $(i, j)_{2 e},(i, j+e)_{2 e},(i+e, j)_{2 e}$, $(i+e, j+e)_{2 e}$, where $i, j \neq 0$ and $i \neq j$, in $\mathbb{F}_{p^{2 r}}$ are not known in general. It is an open problem to determine those. However, Proposition 4.4 immediately gives us the block intersection numbers of the 2-design $\operatorname{dev}\left(C^{H}\right)$ as well as their multiplicities.

Theorem 4.5. Let $C^{H}$ be a $\left(p^{2 r}, \frac{p^{r}-1}{2}, \frac{p^{r}-3}{2}\right)$ difference family in the additive group of $\mathbb{F}_{p^{2 r}}$ constructed using Theorem 3.2. The associated 2- $\left(p^{2 r}, \frac{p^{r}-1}{2}, \frac{p^{r}-3}{2}\right) \operatorname{design} \operatorname{dev}\left(C^{H}\right)$ has exactly the following block intersection numbers.

If $p^{r}-1 \equiv 0(\bmod 4)$, then

\begin{tabular}{ll} 
block intersection number & multiplicity \\
\hline 0 & $\frac{1}{2}\left(3 p^{6 r}+9 p^{5 r}+p^{4 r}-3 p^{3 r}+2 p^{2 r}\right)$ \\
1 & $\frac{1}{2}\left(p^{6 r}-p^{5 r}-p^{4 r}+p^{3 r}\right)$ \\
$\frac{1}{4}\left(p^{r}-5\right)$ & $\frac{1}{2}\left(p^{4 r}-p^{2 r}\right)$ \\
$\frac{1}{4}\left(p^{r}-1\right)$ & $\frac{1}{2}\left(3 p^{4 r}-3 p^{2 r}\right)$
\end{tabular}.

If $p^{r}-1 \equiv 2(\bmod 4)$, then

\begin{tabular}{ll} 
block intersection number & multiplicity \\
\hline 0 & $\frac{1}{2}\left(3 p^{6 r}+9 p^{5 r}+p^{4 r}-3 p^{3 r}+2 p^{2 r}\right)$ \\
1 & $\frac{1}{2}\left(p^{6 r}-p^{5 r}-p^{4 r}+p^{3 r}\right)$ \\
$\frac{1}{4}\left(p^{r}-3\right)$ & $\frac{1}{2}\left(3 p^{4 r}-3 p^{2 r}\right)$ \\
$\frac{1}{4}\left(p^{r}+1\right)$ & $\frac{1}{2}\left(p^{4 r}-p^{2 r}\right)$
\end{tabular}.


Proof. Let $e=p^{r}+1$. It follows from Proposition 4.1 that the block intersection numbers of $\operatorname{dev}\left(C^{H}\right)$ are exactly 0 and the cyclotomic numbers from Proposition 4.4. We obtain their multiplicities using (4): Every cyclotomic number of order $e$ that equals 0 splits into four cyclotomic numbers of order $2 e$ that equal 0 . Every cyclotomic number of order $e$ that takes the value 1 splits into three cyclotomic numbers of order $2 e$ that equal 0 and one cyclotomic number of order $2 e$ that equals 1 . If $p^{r}-1 \equiv 0(\bmod 4)$, the unique cyclotomic number of order $e$ that equals $p^{r}-2$ splits into one cyclotomic number of order $2 e$ that equals $\frac{1}{4}\left(p^{r}-5\right)$ and three cyclotomic numbers of order $2 e$ that equal $\frac{1}{4}\left(p^{r}-1\right)$. If $p^{r}-1 \equiv 2(\bmod 4)$, then we obtain $\frac{1}{4}\left(p^{r}-3\right)$ three times and $\frac{1}{4}\left(p^{r}+1\right)$ once.

Denote by $n_{e}(N)$ the number of cyclotomic numbers of order $e$ that equal $N$. These numbers were given in (3). By the above argumentation, we obtain the following values for $n_{2 e}(N)$. If $p^{r}-1 \equiv 0(\bmod 4)$, then

$$
\begin{aligned}
n_{2 e}(0) & =4 n_{e}(0)+3 n_{e}(1), & n_{2 e}(1) & =n_{e}(1) \\
n_{2 e}\left(\left(p^{r}-5\right) / 4\right) & =n_{e}\left(p^{r}-2\right), & n_{2 e}\left(\left(p^{r}-1\right) / 4\right) & =3 n_{e}\left(p^{r}-2\right) .
\end{aligned}
$$

If $p^{r}-1 \equiv 2(\bmod 4)$, then

$$
\begin{aligned}
n_{2 e}(0) & =4 n_{e}(0)+3 n_{e}(1), & n_{2 e}(1) & =n_{e}(1) \\
n_{2 e}\left(\left(p^{r}-3\right) / 4\right) & =3 n_{e}\left(p^{r}-2\right), & n_{2 e}\left(\left(p^{r}+1\right) / 4\right) & =n_{e}\left(p^{r}-2\right) .
\end{aligned}
$$

From Proposition 4.1, it follows that we need to multiply these numbers with $\frac{1}{2} p^{2 r}\left(p^{2 r}-1\right)$ to obtain the multiplicities of the respective block intersection numbers. For the block intersection number 0 we additionally need to add $\frac{1}{2} p^{2 r}\left(2 p^{2 r}+2\right)\left(2 p^{2 r}+1\right)$.

Next, we examine the intersection numbers of $\operatorname{dev}\left(E^{H}\right)$, the design associated to the disjoint difference family $E^{H}$ in the Galois ring $\operatorname{GR}\left(p^{2}, r\right)$ from Theorem 3.4. Since the design $\operatorname{dev}\left(E^{H}\right)$ is constructed by letting the additive group of $\operatorname{GR}\left(p^{2}, r\right)$ act on the difference family $E^{H}$, there is a strong connection between differences and block intersection numbers: Let $E_{i}^{H}, E_{j}^{H} \in E^{H}$ be two distinct base blocks of $\operatorname{dev}\left(E^{H}\right)$, and let $d$ be a difference occurring $N_{d}$ times in the multiset $E_{i}^{H}-E_{j}^{H}$. Then, $N_{d}$ is the block intersection number $\left|E_{i}^{H} \cap\left(E_{j}^{H}+d\right)\right|$ of the blocks $E_{i}^{H}$ and $E_{j}^{H}+d$ of $\operatorname{dev}\left(E^{H}\right)$. Hence, to calculate the block intersection number $\left|E_{i}^{H} \cap\left(E_{j}^{H}+d\right)\right|$ we need to calculate the multiplicity $N_{d}$ of $d$ in $E_{i}^{H}-E_{j}^{H}$. We will do exactly this for certain base blocks of $\operatorname{dev}\left(E^{H}\right)$.

Let $\xi$ be a generator of the Teichmüller group $\mathcal{T}^{*}$ and let $\mathcal{T}=\mathcal{T}^{*} \cap\{0\}$. As in Theorem 3.4, we denote by $\mathcal{T}_{S}^{*}$ the subgroup of Teichmüller squares and by $\mathcal{T}_{N}^{*}$ the set of Teichmüller non-squares. Furthermore, we call a coset of type

$$
(1+p \alpha) \mathcal{T}_{S}^{*}
$$

where $\alpha \in \mathcal{T}$, a square coset of $\mathcal{T}_{S}^{*}$, and a coset of type

$$
(1+p \alpha) \mathcal{T}_{N}^{*}=(1+p \alpha) \xi \mathcal{T}_{S}^{*},
$$


where $\alpha \in \mathcal{T}$, a non-square coset of $\mathcal{T}_{S}^{*}$. In the remaining part of this section, we will establish bounds on block intersection numbers of $\operatorname{dev}\left(E^{H}\right)$ that come from the multisets $\Delta \mathcal{T}_{S}^{*}$ and $\mathcal{T}_{S}^{*}-\mathcal{T}_{N}^{*}$. We begin by analyzing the structure of these multisets.

Lemma 4.6. Let $p$ be an odd prime. Using the same notation as above, consider the multisets $\Delta \mathcal{T}_{S}^{*}$ and $\mathcal{T}_{S}^{*}-\mathcal{T}_{N}^{*}$ in the Galois ring $G R\left(p^{2}, r\right)$.

- If $p^{r}-1 \equiv 0(\bmod 4)$, then $\Delta \mathcal{T}_{S}^{*}$ contains $\frac{p^{r}-5}{4}$ square cosets and $\frac{p^{r}-1}{4}$ non-square cosets of $\mathcal{T}_{S}^{*}$, and $\mathcal{T}_{S}^{*}-\mathcal{T}_{N}^{*}$ contains $\frac{p^{r}-1}{4}$ square and non-square cosets of $\mathcal{T}_{S}^{*}$ each.

- If $p^{r}-1 \equiv 2(\bmod 4)$, then $\Delta \mathcal{T}_{S}^{*}$ contains $\frac{p^{r}-3}{4}$ square and non-square cosets of $\mathcal{T}_{S}^{*}$ each, and $\mathcal{T}_{S}^{*}-\mathcal{T}_{N}^{*}$ contains $\frac{p^{r}-3}{4}$ square cosets and $\frac{p^{r}+1}{4}$ non-square cosets of $\mathcal{T}_{S}^{*}$.

Proof. Denote by $\mathcal{I}$ the maximal ideal of $\operatorname{GR}\left(p^{2}, r\right)$. The Teichmüller set $\mathcal{T}$ is a system of representatives of $\operatorname{GR}\left(p^{2}, r\right) / \mathcal{I}$ which is isomorphic to the finite field $\mathbb{F}_{p^{r}}$. Hence, the sets of Teichmüller squares $\mathcal{T}_{S}^{*}$ and Teichmüller non-squares $\mathcal{T}_{N}^{*}$ act in the same way as the respective sets of squares and non-squares in $\mathbb{F}_{p^{r}}$. The result now follows from Lemma 4.3: Let $d=s-s^{\prime}$ be the difference of two distinct nonzero squares $s, s^{\prime}$ in $\mathbb{F}_{p^{r}}$. Equivalently,

$$
s d^{-1}-s^{\prime} d^{-1}=1 \text {. }
$$

Note that $d^{-1}$ is a square if and only if $d$ is a square. Using the notation from Lemma 4.3, the equation $s-s^{\prime}=d$ has $Q Q$ solutions for $s, s^{\prime}$ if $d$ is a square, and $N N$ solutions if $d$ is a non-square. Analogously, we obtain the number of solutions for $s, n$ of $s-n=d$, where $s$ is a nonzero square and $n$ is a non-square in $\mathbb{F}_{p^{r}}$.

Furthermore, we need the following properties of squares and non-squares in the Galois $\operatorname{ring} \operatorname{GR}\left(p^{2}, r\right)$.

Proposition 4.7. Consider the Galois ring $G R\left(p^{2}, r\right)$, where $p$ is odd. Denote by $\mathcal{T}_{S}^{*}$ the set of Teichmüller squares and by $\mathcal{T}_{N}^{*}$ the set of Teichmüller non-squares.

1. If $p^{r}-1 \equiv 0(\bmod 4)$, then -1 is a Teichmüller square, and $\mathcal{T}_{S}^{*}=-\mathcal{T}_{S}^{*}$ and $2 \mathcal{T}_{S}^{*} \subseteq \Delta \mathcal{T}_{S}^{*}$.

If $p^{r}-1 \equiv 2(\bmod 4)$, then -1 is a Teichmüller non-square, and $\mathcal{T}_{S}^{*}=-\mathcal{T}_{N}^{*}$ and $2 \mathcal{T}_{S}^{*} \subseteq \mathcal{T}_{S}^{*}-\mathcal{T}_{N}^{*}$

2. If $p^{r}-1 \equiv 0(\bmod 12)$, then $1 \in \Delta \mathcal{T}_{S}^{*}$, and $\mathcal{T}_{S}^{*} \subseteq \Delta \mathcal{T}_{S}^{*}$.

If $p^{r}-1 \equiv 6(\bmod 12)$, then $1 \in \mathcal{T}_{N}^{*}-\mathcal{T}_{S}^{*}$, and $\mathcal{T}_{S}^{*} \subseteq \mathcal{T}_{N}^{*}-\mathcal{T}_{S}^{*}$.

3. If $p^{r}-1 \equiv 0$ or $6(\bmod 8)$, then 2 is a square, and $2 \mathcal{T}_{S}^{*}$ is a square coset of $\mathcal{T}_{S}^{*}$.

If $p^{r}-1 \equiv 2$ or $4(\bmod 8)$, then 2 is a non-square, and $2 \mathcal{T}_{S}^{*}$ is a non-square coset of $\mathcal{T}_{S}^{*}$.

Proof. Let $\xi$ be a generator of the Teichmüller group $\mathcal{T}^{*}$ in the Galois ring $\operatorname{GR}\left(p^{2}, r\right)$. 
1. The present authors [15] proved that if $p$ is odd, -1 is contained in $\mathcal{T}^{*}$, in particular $-1=\xi^{\frac{1}{2}\left(p^{r}-1\right)}$. The exponent $\frac{1}{2}\left(p^{r}-1\right)$ is even if $p^{r}-1 \equiv 0(\bmod 4)$, then -1 is a square in $\mathcal{T}^{*}$. If $p^{r}-1 \equiv 2(\bmod 4)$, the exponent $\frac{1}{2}\left(p^{r}-1\right)$ is odd, hence -1 is a non-square in $\mathcal{T}^{*}$.

2. If $p^{r}-1 \equiv 0(\bmod 6)$, the equation $x^{6}=1$ has exactly six solutions in the Teichmüller group $\mathcal{T}^{*}$, namely $\xi^{k\left(p^{r}-1\right) / 6}$, where $k \in\{0,1, \ldots, 5\}$. We show that the sum of these elements is 0 . It is easy to see that

$$
\xi^{\left(p^{r}-1\right) / 6} \sum_{k=0}^{5} \xi^{k\left(p^{r}-1\right) / 6}=\sum_{k=0}^{5} \xi^{k\left(p^{r}-1\right) / 6} .
$$

Hence,

$$
\left(\xi^{\left(p^{r}-1\right) / 6}-1\right) \sum_{k=0}^{5} \xi^{k\left(p^{r}-1\right) / 6}=0 .
$$

As we have shown in the proof of Theorem 3.4, the element $\xi^{\left(p^{r}-1\right) / 6}-1$ is a unit. It follows that

$$
\sum_{k=0}^{5} \xi^{k\left(p^{r}-1\right) / 6}=0
$$

By the same reasoning, $\sum_{k=0}^{2} \xi^{k\left(p^{r}-1\right) / 3}=0$. Consequently, we can rewrite (7) as

$$
\xi^{5\left(p^{r}-1\right) / 6}-\xi^{2\left(p^{r}-1\right) / 3}=1 .
$$

If $p^{r}-1 \equiv 0(\bmod 12)$, the elements $\xi^{5\left(p^{r}-1\right) / 6}$ and $\xi^{2\left(p^{r}-1\right) / 3}$ are squares and, consequently, $1 \in \Delta \mathcal{T}_{S}^{*}$. If $p^{r}-1 \equiv 6(\bmod 12)$, then $\xi^{5\left(p^{r}-1\right) / 6}$ is a non-square and $\xi^{2\left(p^{r}-1\right) / 3}$ is a square, hence $1 \in \mathcal{T}_{N}^{*}-\mathcal{T}_{S}^{*}$.

3. We first consider $r=1$. Note that $\operatorname{GR}\left(p^{2}, 1\right)=\mathbb{Z}_{p^{2}}$. The following classical results about quadratic residues were first systematically given by Gauss [13]. An element $a$ relatively prime to an odd prime $p$ is a square in $\mathbb{Z}_{p^{m}}$ if and only if $a$ is a square in $\mathbb{Z}_{p}$. In $\mathbb{Z}_{p}$, the element 2 is a square if $p-1 \equiv 0$ or $6(\bmod 8)$, and 2 is a non-square if $p-1 \equiv 2$ or $4(\bmod 8)$. This solves the problem for $r=1$.

Now, let $r \geq 2$. Let

$$
\mathcal{T}_{1}^{*}=\left\{1, \zeta, \zeta^{2}, \ldots, \zeta^{p-2}\right\}
$$

denote the Teichmüller group of $\operatorname{GR}\left(p^{2}, 1\right)$, and let $\mathcal{T}_{1}=\mathcal{T}_{1}^{*} \cup\{0\}$. For a fixed prime $p$, the Galois ring $\operatorname{GR}\left(p^{2}, 1\right)$ is a subring of $\operatorname{GR}\left(p^{2}, r\right)$ for all $r \geq 1$. If $\mathcal{T}^{*}=\langle\xi\rangle$ denotes the Teichmüller group of $\operatorname{GR}\left(p^{2}, r\right)$, then $\mathcal{T}_{1}^{*}$ is a subgroup of $\mathcal{T}^{*}$ and we write

$$
\mathcal{T}_{1}^{*}=\left\{1, \xi^{\left(p^{r}-1\right) /(p-1)}, \xi^{2\left(p^{r}-1\right) /(p-1)}, \ldots, \xi^{(p-2)\left(p^{r}-1\right) /(p-1)}\right\},
$$


where $\zeta^{k}=\xi^{k\left(p^{r}-1\right) /(p-1)}$. Since 2 is a unit in $\operatorname{GR}\left(p^{2}, 1\right)$, we write $2=\left(1+p \alpha_{0}\right) \alpha_{1}$ for unique $\alpha_{0}, \alpha_{1}$, where $\alpha_{0} \in \mathcal{T}_{1}$ and $\alpha_{1} \in \mathcal{T}_{1}^{*}$. It follows that $\alpha_{1}=\zeta^{\ell}$ for some $\ell \in\{0,1, \ldots, p-2\}$. In $\operatorname{GR}\left(p^{2}, r\right)$, we consequently obtain

$$
2=\left(1+p \alpha_{0}\right) \xi^{\ell\left(p^{r}-1\right) /(p-1)} .
$$

Hence, 2 is a square, and thereby $2 \mathcal{T}_{S}^{*}$ is a square coset of $\mathcal{T}_{S}^{*}$, if at least one of the two numbers $\ell$ and $\left(p^{r}-1\right) /(p-1)$ is even. The second number is even if and only if $r$ is even. In this case, $p^{r}-1 \equiv 0(\bmod 8)$. Hence, if $r$ is odd, the number $\ell$ needs to be even. This is the case if and only if 2 is a square in $\operatorname{GR}\left(p^{2}, 1\right)$, which, according to the case $r=1$, holds whenever $p-1 \equiv 0$ or $6(\bmod 8)$. If $r$ is odd, $p^{r} \equiv p(\bmod 8)$. The result follows.

By combining all three results from Proposition 4.7, we obtain the following corollary:

Corollary 4.8. Consider the Galois ring $G R\left(p^{2}, r\right)$, where $p$ is odd. Denote by $\mathcal{T}_{S}^{*}$ the set of Teichmüller squares and by $\mathcal{T}_{N}^{*}$ the set of Teichmüller non-squares.

- If $p^{r}-1 \equiv 0(\bmod 12)$, then the multiset $\Delta \mathcal{T}_{S}^{*}$ contains both $\mathcal{T}_{S}^{*}$ and $2 \mathcal{T}_{S}^{*}$, and the set $2 \mathcal{T}_{S}^{*}$ is a square coset of $\mathcal{T}_{S}^{*}$ if and only if $p^{r}-1 \equiv 0(\bmod 24)$.

- If $p^{r}-1 \equiv 6(\bmod 12)$, then the multiset $\mathcal{T}_{S}^{*}-\mathcal{T}_{N}^{*}$ contains both $\mathcal{T}_{N}^{*}$ and $2 \mathcal{T}_{S}^{*}$, and the set $2 \mathcal{T}_{S}^{*}$ is a non-square coset of $\mathcal{T}_{S}^{*}$ if and only if $p^{r}-1 \equiv 18(\bmod 24)$.

Note that $p^{r}-1 \equiv 0(\bmod 24)$ holds whenever the prime $p \geq 5$ and $r$ is even. To continue, we need the following result about when 2 is a Teichmüller square.

Lemma 4.9. Consider the Galois ring $G R\left(p^{2}, r\right)$, where $p$ is odd. Denote by $\mathcal{T}_{S}^{*}$ the set of Teichmüller squares and by $\mathcal{T}_{N}^{*}$ the set of Teichmüller non-squares.

- If both $p^{r}-1 \equiv 0$ or $6(\bmod 8)$ and $2^{p-1} \equiv 1\left(\bmod p^{2}\right)$, then $\mathcal{T}_{S}^{*}=2 \mathcal{T}_{S}^{*}$.

- If both $p^{r}-1 \equiv 2$ or $4(\bmod 8)$ and $2^{p-1} \equiv 1\left(\bmod p^{2}\right)$, then $\mathcal{T}_{N}^{*}=2 \mathcal{T}_{S}^{*}$.

Proof. The equation $\mathcal{T}_{S}^{*}=2 \mathcal{T}_{S}^{*}$ holds if and only if 2 is a square in the Teichmüller group $\mathcal{T}^{*}$. According to Proposition 4.7, the element 2 is a square in $\operatorname{GR}\left(p^{2}, r\right)$ if and only if $p^{r}-1 \equiv 0$ or $6(\bmod 8)$. Since $\mathcal{T}^{*}$ has order $p^{r}-1$, the element 2 is contained in $\mathcal{T}^{*}$ if and only if $2^{p^{r}-1} \equiv 1\left(\bmod p^{2}\right)$. Since 2 is also an element of $\mathbb{Z}_{p^{2}}=\operatorname{GR}\left(p^{2}, 1\right)$ which is a subring of $\operatorname{GR}\left(p^{2}, r\right)$, this condition can be reduced to $2^{p-1} \equiv 1\left(\bmod p^{2}\right)$.

On the other hand, the equation $\mathcal{T}_{N}^{*}=2 \mathcal{T}_{S}^{*}$ holds if and only if 2 is a non-square and $2 \in \mathcal{T}^{*}$. The second statement now follows by analogous reasoning as above from Proposition 4.7.

Primes that solve $2^{p-1} \equiv 1\left(\bmod p^{2}\right)$ are called Wieferich primes. So far, the only known Wieferich primes are 1093 and 3511. Thus, the only known Galois rings of characteristic $p^{2}$ satisfying $\mathcal{T}_{S}^{*}=2 \mathcal{T}_{S}^{*}$ are $\operatorname{GR}\left(1093^{2}, r\right)$, where $r$ is even, and $\operatorname{GR}\left(3511^{2}, r\right)$ for arbitrary $r$. The only known Galois ring of characteristic $p^{2}$ satisfying $\mathcal{T}_{N}^{*}=2 \mathcal{T}_{S}^{*}$ is $\operatorname{GR}\left(1093^{2}, r\right)$, where $r$ is odd. 
With the help of the first result given in Proposition 4.7, we now establish a lower bound on the multiplicities of certain differences of Teichmüller elements. This is an analogue of the present authors' previous result [15, Lemma 5.9] for the design $\operatorname{dev}(C)$ from Theorem 3.3:

Lemma 4.10. Consider the Galois ring $G R\left(p^{2}, r\right)$, where $p$ is odd. Denote by $\mathcal{T}_{S}^{*}$ the set of Teichmüller squares and by $\mathcal{T}_{N}^{*}$ the set of Teichmüller non-squares.

- If $p^{r}-1 \equiv 0(\bmod 4)$, then all differences $d \in \Delta \mathcal{T}_{S}^{*}$ where $d \notin 2 \mathcal{T}_{S}^{*}$ have multiplicity $N_{d}>1$ in $\Delta \mathcal{T}_{S}^{*}$.

- If $p^{r}-1 \equiv 2(\bmod 4)$, then all differences $d \in \mathcal{T}_{S}^{*}-\mathcal{T}_{N}^{*}$ where $d \notin 2 \mathcal{T}_{S}^{*}$ have mulitplicity $N_{d}>1$ in $\mathcal{T}_{S}^{*}-\mathcal{T}_{N}^{*}$.

Proof. We prove the first result. The proof of the second statement is analogous. Let $p$ be a prime and $r$ be a positive integer such that $p^{r}-1 \equiv 0(\bmod 4)$. Moreover, let $d \in \Delta \mathcal{T}_{S}^{*}$, which means that $d=s-s^{\prime}$ is the difference of two distinct Teichmüller squares $s, s^{\prime} \in \mathcal{T}_{S}^{*}$. According to Proposition $4.7, \mathcal{T}_{S}^{*}=-\mathcal{T}_{S}^{*}$. Hence, if $s^{\prime} \neq s$, then $\left(-s^{\prime}\right)-(-s)=d$ is a second representation of $d$ in $\Delta \mathcal{T}_{S}^{*}$. Note that all these differences occur in pairs. If $s^{\prime}=-s$, however, the two representations are the same, and $d=2 s$, thus $d \in 2 \mathcal{T}_{S}^{*}$. The statement follows.

In the following lemma, we will establish an upper bound on the multiplicity of certain differences in $\Delta \mathcal{T}_{S}^{*}$ and $\mathcal{T}_{S}^{*}-\mathcal{T}_{N}^{*}$. For our main theorem, only the first part of the lemma is relevant. However, we also state the second part as it is easily obtained from the previous results.

Lemma 4.11. Let $p$ be an odd prime such that $2^{p-1} \not \equiv 1\left(\bmod p^{2}\right)$. Consider the Galois ring $G R\left(p^{2}, r\right)$, and denote by $\mathcal{T}_{S}^{*}$ the set of Teichmüller squares and by $\mathcal{T}_{N}^{*}$ the set of Teichmüller non-squares.

- If $p^{r}-1 \equiv 0(\bmod 24)$, then all differences $d \in \Delta \mathcal{T}_{S}^{*}$ where $d$ is a square have multiplicity $N_{d}<\frac{p^{r}-5}{4}$ in $\Delta \mathcal{T}_{S}^{*}$

- If $p^{r}-1 \equiv 18(\bmod 24)$, then all differences $d \in \mathcal{T}_{S}^{*}-\mathcal{T}_{N}^{*}$ have multiplicity $N_{d}<$ $\frac{p^{r}+1}{4}$ in $\mathcal{T}_{S}^{*}-\mathcal{T}_{N}^{*}$.

Proof. The condition $2^{p-1} \not \equiv 1\left(\bmod p^{2}\right)$ ensures that $2 \notin \mathcal{T}^{*}$, and consequently $\mathcal{T}_{S}^{*} \neq$ $2 \mathcal{T}_{S}^{*} \neq \mathcal{T}_{N}^{*}$ as we showed in Lemma 4.9. Assume $p^{r}-1 \equiv 0(\bmod 24)$, and let $d$ be the difference of two Teichmüller squares, $d \in \Delta \mathcal{T}_{S}^{*}$, such that $d$ is a square. Denote by $N_{d}$ the multiplicity of $d$ in $\Delta \mathcal{T}_{S}^{*}$. From Lemma 4.6, we know that $\Delta \mathcal{T}_{S}^{*}$ contains $\frac{1}{4}\left(p^{r}-5\right)$ not necessarily distinct square cosets of $\mathcal{T}_{S}^{*}$. It follows that $N_{d} \leq \frac{1}{4}\left(p^{r}-5\right)$, and $N_{d}=\frac{1}{4}\left(p^{r}-5\right)$ if and only if $\Delta \mathcal{T}_{S}^{*}$ contains only exactly one square coset of $\mathcal{T}_{S}^{*}$ with multiplicity $\frac{1}{4}\left(p^{r}-5\right)$. Assume $N_{d}=\frac{1}{4}\left(p^{r}-5\right)$. If $p^{r}-1 \equiv 0(\bmod 24)$, then, according to Corollary 4.8 , both $\mathcal{T}_{S}^{*}$ and $2 \mathcal{T}_{S}^{*}$ are subsets of $\Delta \mathcal{T}_{S}^{*}$, and $2 \mathcal{T}_{S}^{*}$ is a square coset of $\mathcal{T}_{S}^{*}$. This is a contradiction. 
Now, assume $p^{r}-1 \equiv 18(\bmod 24)$, and let $d$ be the difference of a Teichmüller square and a Teichmüller non-square, $d \in \mathcal{T}_{S}^{*}-\mathcal{T}_{N}^{*}$, such that $d$ is a non-square. Denote by $N_{d}$ the multiplicity of $d$ in $\mathcal{T}_{S}^{*}-\mathcal{T}_{N}^{*}$. Analogously to above, we conclude from Lemma 4.6 that $N_{d} \leq \frac{1}{4}\left(p^{r}+1\right)$, and $N_{d}=\frac{1}{4}\left(p^{r}+1\right)$ if and only if $\mathcal{T}_{S}^{*}-\mathcal{T}_{N}^{*}$ contains only exactly one non-square coset of $\mathcal{T}_{S}^{*}$. Assume $N_{d}=\frac{1}{4}\left(p^{r}+1\right)$. If $p^{r}-1 \equiv 18(\bmod 24)$, then both $\mathcal{T}_{N}^{*}$ and $2 \mathcal{T}_{S}^{*}$ are contained in $\mathcal{T}_{S}^{*}-\mathcal{T}_{N}^{*}$, and $2 \mathcal{T}_{S}^{*}$ is a non-square coset of $\mathcal{T}_{S}^{*}$. Again, we obtain a contradiction.

As we have mentioned above, the multiplicity of a difference $d$ in $\Delta \mathcal{T}_{S}$ corresponds directly to the block intersection number $\left|\mathcal{T}_{S}^{*} \cap\left(\mathcal{T}_{S}^{*} \cap d\right)\right|$. Hence, we obtain from the previous lemmas the following theorem which is our main theorem.

Theorem 4.12. Let $p$ be an odd prime such that $2^{p-1} \not \equiv 1\left(\bmod p^{2}\right)$. Let $C^{H}$ be a $\left(p^{2 r}, \frac{p^{r}-1}{2}, \frac{p^{r}-3}{2}\right)$ disjoint difference family in the additive group of the finite field $\mathbb{F}_{p^{2 r}}$ constructed with Theorem 3.2, and let $E^{H}$ be a disjoint difference family with the same parameters in the additive group of the Galois ring $G R\left(p^{2}, r\right)$ constructed with Theorem 3.4. If $p^{r}-1 \equiv 0(\bmod 24)$, the $2-\left(p^{2 r}, \frac{p^{r}-1}{2}, \frac{p^{r}-3}{2}\right) \operatorname{designs} \operatorname{dev}\left(E^{H}\right)$ and $\operatorname{dev}\left(C^{H}\right)$ are nonisomorphic.

Proof. Let $p$ be an odd prime and $r$ be an integer such that $p^{r}-1 \equiv 0(\bmod 24)$. Recall from Theorem 4.5 that in this case the block intersection numbers of our design $\operatorname{dev}\left(C^{H}\right)$ are given as $0,1, \frac{1}{4}\left(p^{r}-5\right), \frac{1}{4}\left(p^{r}-1\right)$. Now, consider the Galois ring $\operatorname{GR}\left(p^{2}, r\right)$, and denote by $\mathcal{T}_{S}^{*}$ the set of Teichmüller squares and by $\mathcal{T}_{N}^{*}$ the set of Teichmüller non-squares. By combining Lemma 4.10 and Lemma 4.11, we obtain

$$
1<\left|\mathcal{T}_{S}^{*} \cap\left(\mathcal{T}_{S}^{*}+d\right)\right|<\frac{p^{r}-5}{4}
$$

for all squares $d \in \Delta \mathcal{T}_{S}^{*} \backslash 2 \mathcal{T}_{S}^{*}$. Consequently, the design $\operatorname{dev}\left(E^{H}\right)$ has an intersection number different from the ones of $\operatorname{dev}\left(C^{H}\right)$, and the designs are nonisomorphic.

We remark that $p^{r}-1 \equiv 0(\bmod 24)$ holds for all $p$ and $r$ where $p \geq 5$ and $r$ is even. Furthermore, we remark that for the Wieferich primes 1093 and 3511, that satisfy $2^{p-1} \equiv 1\left(\bmod p^{2}\right)$, the designs $\operatorname{dev}\left(C^{H}\right)$ and $\operatorname{dev}\left(E^{H}\right)$ are nonisomorphic for all $r>1$. With the help of Magma [4] we computed the multisets $\Delta \mathcal{T}_{S}^{*}$ and $\mathcal{T}_{S}^{*}-\mathcal{T}_{N}^{*}$ for $r=1$ and checked that these multisets contain more than one square coset and more than two non-square coset of $\mathcal{T}_{S}^{*}$ each. Consequently, there will be at least as many square and non-square cosets of $\mathcal{T}_{S}^{*}$ in $\Delta \mathcal{T}_{S}^{*}$ and $\mathcal{T}_{S}^{*}-\mathcal{T}_{N}^{*}$, respectively, for $r>1$. So, the bounds on the respective block intersection numbers established in the previous proof hold.

To conclude this section, we give an example that demonstrates why our block intersection number approach fails if $p^{r}-1 \not \equiv 0(\bmod 24)$. We choose as an example the case $p^{r}-1 \equiv 18(\bmod 24)$ since, in the previous lemmas, we have already obtained several results about this case that followed immediately from the results for $p^{r}-1 \not \equiv 0$ $(\bmod 24)$. 
Example 3. Let $p$ and $r$ such that $p^{r}-1 \equiv 18(\bmod 24)$. In this case, according to Theorem 4.5, the design $\operatorname{dev}\left(C^{H}\right)$, has block intersection numbers $0,1, \frac{1}{4}\left(p^{r}-3\right)$, $\frac{1}{4}\left(p^{r}+1\right)$. For the design $\operatorname{dev}\left(E^{H}\right)$, using Lemma 4.10 and Lemma 4.11, we obtain

$$
1<\left|\mathcal{T}_{S}^{*} \cap\left(\mathcal{T}_{N}^{*}+d\right)\right|<\frac{p^{r}+1}{4}
$$

for all $d \in\left(\mathcal{T}_{S}^{*}-\mathcal{T}_{N}^{*}\right) \backslash 2 \mathcal{T}_{S}^{*}$. However, this result is of little use as it is still possible that there exists $d \in\left(\mathcal{T}_{S}^{*}-\mathcal{T}_{N}^{*}\right) \backslash 2 \mathcal{T}_{S}^{*}$ such that $\left|\mathcal{T}_{S}^{*} \cap\left(\mathcal{T}_{N}^{*}+d\right)\right|=\frac{1}{4}\left(p^{r}-3\right)$ or that two completely different blocks intersect in $\frac{1}{4}\left(p^{r}+1\right)$ elements. In fact, the multiset $p \mathcal{T}_{S}^{*}-p \mathcal{T}_{N}^{*}$ contains $\frac{1}{4}\left(p^{r}+1\right)$ times the set $p \mathcal{T}_{N}^{*}$ and $\frac{1}{4}\left(p^{r}-3\right)$ times the set $p \mathcal{T}_{S}^{*}$. Hence, these two numbers actually occur as the block intersection numbers

$$
\left|p \mathcal{T}_{S}^{*} \cap\left(p \mathcal{T}_{N}^{*}+d\right)\right|,
$$

where $d \in \mathcal{I} \backslash\{0\}$. Hence, we cannot show the existence of an intersection number $N$ such that $1<N<\frac{1}{4}\left(p^{r}-3\right)$.

\section{Conclusion and open questions}

Motivated by the present authors' [15] recent results, we tried to use the same technique to solve another isomorphism problem about $2-(v, k, k-1)$ designs. Thanks to the algebraic structure of our designs, we were able to solve the problem for many cases, and, in doing so, obtained some interesting results about cyclotomic numbers and the structure of Galois rings of characteristic $p^{2}$. But the isomorphism problem is still not solved for all cases.

Our results demonstrate that using block intersection numbers as a method to tackle isomorphism problems about combinatorial designs has its limitations. One needs designs that have a sufficiently strong algebraic structure to calculate or even bound these numbers. We still consider this approach promising, especially if the designs are constructed as the developments of some difference structures. During our studies, we discovered the following interesting open problems:

- Our computations hint that Theorem 4.12 holds for all $p$ and $r$, where $p$ is odd. However, our examination of intersection numbers did not lead to the results necessary to prove this conjecture. We leave this task to future work.

- The construction of a disjoint difference family in $\operatorname{GR}\left(p^{2}, r\right)$ presented in Theorem 3.4 does not only work for the subgroup of squares in the Teichmüller group but for all its subgroups. Moreover, there will always be an analogue in $\mathbb{F}_{p^{2 r}}$. It would be interesting to study the isomorphism problem for the associated designs in all these cases. It might be possible to deduce more block intersection numbers from the ones given in this paper and in [15].

- As mentioned before, nonisomorphic designs can have the same block intersection numbers. It would be interesting to find more difference families as in Remark 1 for which their associated designs have the same intersection numbers but are still nonisomorphic. 


\section{References}

\section{References}

[1] R. J. R. Abel and M. Buratti. Difference families. In: Handbook of Combinatorial Designs. Ed. by C. J. Colbourn and J. H. Dinitz. 2nd ed. Boca Raton: Chapman \& Hall/CRC Press, 2007, pp. 392-410.

[2] L. D. Baumert, W. H. Mills, and R. L. Ward. Uniform cyclotomy. In: J. Number Theory 14.1 (1982), pp. 67-82.

[3] T. Beth, D. Jungnickel, and H. Lenz. Design Theory. 2nd ed. Cambridge: Cambridge University Press, 1999.

[4] W. Bosma, J. Cannon, and C. Playoust. The Magma algebra system. I. The user language. In: J. Symbolic Comput. 24.3-4 (1997), pp. 235-265.

[5] M. Buratti. On disjoint $(v, k, k-1)$ difference families. In: Des. Codes Cryptogr. Special Issue: Finite Geometries (2018).

[6] M. Buratti and D. Jungnickel. Partitioned difference families versus zero-difference balanced functions. In: Des. Codes Cryptogr. (2019), pp. 1-7.

[7] Y. Chang and C. Ding. Constructions of external difference families and disjoint difference families. In: Des. Codes Cryptogr. 40.2 (2006), pp. 167-185.

[8] C. J. Colbourn and J. H. Dinitz, eds. Handbook of Combinatorial Designs. 2nd ed. Boca Raton: Chapman \& Hall/CRC Press, 2007.

[9] J. A. Davis, S. Huczynska, and G. L. Mullen. Near-complete external difference families. In: Des. Codes Cryptogr. 84 (2017), pp. 415-424.

[10] L. E. Dickson. Linear Groups: With an Exposition of the Galois Field Theory. New York: Dover Publications, 1958.

[11] T. Feng and Q. Xiang. Cyclotomic constructions of skew hadamard difference sets. In: J. Comb. Theory, Ser. A 119.1 (2012), pp. 245-256.

[12] S. Furino. Difference families from rings. In: Discrete Math. 97.1-3 (1991), pp. 177190.

[13] C.F. Gauss. Untersuchungen über höhere Arithmetik. Trans. by H. Maser. New York: Chelsea Publishing Company, 1981.

[14] J. Jedwab and S. Li. Construction and nonexistence of strong external difference families. In: J. Alg. Comb. 49.1 (2019), pp. 21-48. 


\section{References}

[15] C. Kaspers and A. Pott. Solving isomorphism problems about 2-designs from disjoint difference families. In: J. Combin. Des. 27.5 (2019), pp. 277-294.

[16] B. R. McDonald. Finite Rings with Identity. New York: Marcel Dekker, Inc., 1974.

[17] K. Momihara. Disjoint difference families from Galois rings. In: Electron. J. Combin. 24.3 (2017), P3.23.

[18] S.-L. Ng and M. B. Paterson. Disjoint difference families and their applications. In: Des. Codes Cryptogr. 78.1 (2016), pp. 103-127.

[19] T. Ralston. On the distribution of squares in a finite field. In: Geom. Dedicata 8.2 (1979), pp. 207-212.

[20] Z.-X. Wan. Lectures on Finite Fields and Galois Rings. Singapore: World Scientific, 2003.

[21] R. M. Wilson. Cyclotomy and difference families in elementary abelian groups. In: J. Number Theory 4.1 (1972), pp. 17-47.

[22] J. Zwanzger. Computergestützte Suche nach optimalen linearen Codes über endlichen Kettenringen unter Verwendung heuristischer Methoden. PhD thesis. Bayreuth: University of Bayreuth, 2011. 